\title{
FORMAÇÕES FLORESTAIS DE RESTINGA E RELAÇÕES ECOLÓGICAS COM LIANAS $^{1}$
}

\begin{abstract}
Adriano Costa Quaresma² e Mário Augusto Gonçalves Jardim³
RESUMO - Objetivou-se conhecer a composição florística das lianas em duas formações florestais do litoral amazônico paraense. O estudo foi realizado na APA Algodoal-Maiandeua, no Município de Maracanã, Pará, onde foram demarcadas duas parcelas de 50 x $50 \mathrm{~m}$ na floresta seca e duas parcelas na floresta inundável. Para análise da composição, riqueza e diversidade, foram identificadas e quantificadas todas as espécies, enquanto para verificar a diferença entre as comunidades de trepadeiras foram sorteadas aleatoriamente 80 árvoressuporte em cada formação florestal e utilizada uma análise de MDS. A diversidade foi verificada pelos índices de Shannon-Weaver (H') e o inverso, pelo índice de Simpson (1/D). Nas duas formações foram registrados 177 indivíduos, em 15 famílias, 21 gêneros e 27 espécies. Dilleniaceae foi a família com o maior número de espécies e Smilax syphilitica Humb. \& Bonpl. ex Willd. e Odontadenia nitida (Vahl) Müll. Arg., com o maior número de indivíduos na floresta seca e na floresta inundável, respectivamente. A análise de MDS não separou as florestas de acordo com a composição florística das lianas. A diversidade na floresta inundável foi de $H^{\prime}=1,08$ e $1 / D=8,3$ e na floresta seca, de $H^{\prime}=0,98$ e 1/D = 7,2. As formações florestais analisadas não apresentaram diferenças significativas quanto à diversidade, riqueza e composição de lianas.
\end{abstract}

Palavras-chave: Diversidade; Riqueza; Litoral amazônico.

\section{COASTAL FOREST FORMATIONS AND ECOLOGICAL RELATIONSHIPSWITH VINES}

\begin{abstract}
The aim of this study was to know the floristic composition of vines in forest formations at the coast of Amazon. The study took place in the Algodoal-Maiandeua Area Environmental Protection, Maracanã city, Pará State, where two 50x50m parcels were demarcated in a dry forest and two in a flooding forest. For the analysis of composition, richness and diversity all the species were identified and quantified and to verify the differences between the two vines communities in 80 support trees were randomly selected in each forest formation and then a MDS analysis was done. The diversity was measured using the ShannonWeaver ( $\left.H^{\prime}\right)$ and the inverse Simpson Index (1/D). In the two forest formations, 177 individuals were registered, in 15 families, 21 genera and 27 species. Dilleniaceae was the family with the greater number of species and Smilax syphilitica Humb. \& Bonpl. ex Willd. e Odontadenia nitida (Vahl) Müll. Arg. the greater number of individuals in the dry forest and flooding forest, respectively. The MDS analysis did not group the forests by their floristic composition. The diversity at the flooding forest was $H^{\prime}=1.08$ and $1 / \mathrm{D}=8.3$, for the dry forest it was $H^{\prime}=0.98$ and $1 / D=7.2$. The type of coastal forest formation does not influence the creepers/ vines community composition, richness and diversity.
\end{abstract}

Keywords: Diversity; Richness; Amazon coast.

\footnotetext{
${ }^{1}$ Recebido em 15.07.2014 aceito para publicação em 15.12.2014.

${ }^{2}$ Universidade Federal Rural da Amazônia, Programa de Pós-Graduação em Ciências Biológicas. E-mail: <acq.quaresma@gmail.com>. ${ }^{3}$ Ministério da Ciência, Tecnologia e Inovação, Museu Paraense Emílio Goeldi, Belem, PA - Brasil. E-mail: <jardim@museugoeldi.br>.
}

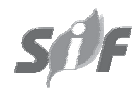

Revista Árvore, Viçosa-MG, v.39, n.2, p.255-261, 2015

http://dx.doi.org/10.1590/0100-67622015000200005 


\section{INTRODUÇÃO}

Lianas são plantas lenhosas ou herbáceas, cujas sementes germinam e, ao se desenvolverem, mantêm contato com o solo durante todo o seu ciclo de vida. Seu crescimento em altura é condicionado por outras plantas que servem como suporte (QUARESMA; JARDIM, 2014). Para Brandes e Barros (2008), durante o crescimento perdem sua habilidade de se sustentar, necessitando de suporte para ascender à copa. São abundantes e diversificadas, contudo, sua riqueza nas florestas tropicais é maior do que nas florestas temperadas (SCHMITZER; BONGERS, 2002).

$\mathrm{Na}$ composição florestal, as lianas possuem alta diversidade em formas e adaptações e nas florestas tropicais úmidas ou florestas subtropicais com ausência de perturbações chegam a contribuir com 5\% do total de biomassa (VILLAGRA; NETO, 2010). Na dinâmica florestal, desempenham papel vital que inclui a supressão da regeneração e do crescimento em diâmetro e favorece o aumento da mortalidade das árvores que as suportam devido ao seu peso excessivo (CARVALHO et al., 2013). Apresentam rápido crescimento quando comparadas com as espécies arbóreas e chegam a dominar a copa do seu forófito (HORA; SOARES, 2002).

Por esse motivo, as lianas exercem importante papel na colonização e competição de árvores e, consequentemente, na composição global da comunidade arbórea (SCHMITZER; BONGERS, 2002). Adependência de substrato arbóreo em associação com a sensibilidade à umidade fazem com que atuem como indicadores ecológicos da qualidade do ambiente e das variações ambientais naturais (FREITAS; ASSIS, 2013).

No Brasil, os inventários florísticos pouco têm levado em consideração o componente lianas. Nas florestas brasileiras há evidente escassez de trabalhos, tornando-se necessárias mais investigações tanto da florística quanto dos aspectos ecológicos do grupo. Nesse contexto, alguns estudos foram realizados na floresta ombrófila densa (CITADINI-ZANETTE et al., 1997), na floresta estacional semidecidual (MORELLATO; LEITÃO-FILHO, 1998; HORA; SOARES, 2002; DURIGON et al., 2009) e na floresta de terra firme amazônica (OLIVEIRA et al., 2008). Esses estudos registraram as maiores riquezas para Bignoniaceae, Convolvulaceae, Malpighiaceae, Sapindaceae e Apocynaceae.

O conhecimento restrito sobre a florística de lianas é agravado pela dificuldade de visualização e coleta, pois ocupam o dossel florestal. Dessa forma, levantamentos florísticos que registram todas as formas de vida geralmente excluem grande parte das espécies escandentes (GENTRY, 1991). Isso ressalta a importância da amostragem florística direcionada e intensificada para o grupo, que é o primeiro passo para a realização de um manejo bem-sucedido (TIBIRIÇA et al., 2006).

Este trabalho objetivou avaliar a composição, riqueza e diversidade de lianas em duas formações florestais de restinga na Área de Proteção Ambiental, AlgodoalMaiandeua, Pará, Brasil.

\section{MATERIAL E MÉTODOS}

A Área de Proteção Ambiental Algodoal-Maiandeua está localizada no Município de Maracanã, no litoral Nordeste do Estado do Pará sob as coordenadas geográficas 00³8'29"S e 47³1'54" W. A região apresenta clima tropical úmido do tipo Awi, da classificação de Köppen, com temperatura média máxima anual de 31,7 ${ }^{\circ} \mathrm{C}$ e mínima anual de $25,2{ }^{\circ} \mathrm{C}$, apresentando valor médio anual de índice pluviométrico em torno $2.800 \mathrm{~mm}$ (JARDIM et al., 2013).

As formações florestais foram descritas com base em Amaral et al. (2008) e Jardim et al. (2013) como: a) Floresta seca - apresenta nível topográfico de pouco plano a ondulado; solo muito arenoso, com pH igual a 4,95, teor de matéria orgânica $\left(69,46 \mathrm{~g} \cdot \mathrm{kg}^{-1}\right)$, concentrações de fósforo $\left(12,58 \mathrm{mg} \cdot \mathrm{dm}^{-3}\right)$, de nitrogênio $\left(0,83 \mathrm{~g} \cdot \mathrm{kg}^{-1}\right)$ e de ferro $\left(69,5 \mathrm{mg} \cdot \mathrm{dm}^{-3}\right)$; luminosidade regular; biomassa foliar elevada e com a dominância de Anacardium occidentale L., Pouteria ramiflora (Mart.) Radlk., Pradosia schomburgkiana (A.DC.) Cronquist e Myrcia rufipila McVaugh; e b) Floresta inundável - com topografia plana com algumas depressões inundadas, principalmente no período chuvoso na região; solo pouco arenoso, com $\mathrm{pH}$ igual a 4,36, teor de matéria orgânica $\left(57,67 \mathrm{~g} \cdot \mathrm{kg}^{-1}\right)$, concentrações de fósforo $\left(2,58 \mathrm{mg} \cdot \mathrm{dm}^{-3}\right)$, de nitrogênio $\left(0,83 \mathrm{~g} \cdot \mathrm{kg}^{-1}\right)$ e de ferro $\left(7,5 \mathrm{mg} \cdot \mathrm{dm}^{-3}\right)$; muito sombreada, com pouca incidência luminosa; biomassa foliar elevada e com a dominância de Virola surinamensis (Rol. Ex Rottb.) Warb., Symphonia globulifera L.f., Macrolobium pendulum Willd. ex Vogel e Euterpe oleracea Mart.

Foram demarcadas duas parcelas de $50 \times 50 \mathrm{~m}$ na floresta seca (P1 - 00 $35^{\circ} 15,2^{\prime \prime} \mathrm{S}$ e $47^{\circ} 34^{\prime} 21^{\prime \prime} \mathrm{W}$; P2 $00^{\circ} 35^{\prime} 8,4^{\prime \prime S}$ e $\left.47^{\circ} 34^{\prime} 24,7^{\prime \prime} \mathrm{W}\right)$, duas parcelas na floresta inundável (P3 - 00³6’34,1" S e 47³4'39,3" W e P4

Revista Árvore, Viçosa-MG, v.39, n.2, p.255-261, 2015 
$-00^{\circ} 36^{\prime} 35,5^{\prime \prime} \mathrm{S}$ e $47^{\circ} 34^{\prime} 34,2^{\prime \prime} \mathrm{W}$ ) e registradas todas as trepadeiras nas árvores que apresentaram DAP $\geq$ $5 \mathrm{~cm}$.

As amostras de espécies de trepadeiras e de árvoressuporte foram coletadas e herborizadas segundo as técnicas consagradas em sistemática vegetal e sempre que possível foram identificadas in loco ou posteriormente por comparação com o acervo do Herbário João Murça Pires (MG - Museu Paraense Emilio Goeldi). Foram utilizados, quando necessários, binóculos e escaladas livres, para melhor visualização e contagem das trepadeiras.

Para verificar a diferença entre as comunidades de trepadeiras das duas formações florestais, foram sorteadas aleatoriamente 80 árvores-suporte dentro de cada parcela alocada em cada tipo de formação florestal, que foram utilizadas como unidades amostrais. Posteriormente, foi realizada uma técnica de ordenação através da análise de escalonamento mutidimensional não métrica (MDS). Para isso, foram construídas matrizes de similaridade utilizando o índice de BrayCurtis como medida de distância, com base nos dados de abundância, transformados em raiz-quadrada, buscando reduzir a influência de espécies dominantes. A análise de ordenação foi realizada com o aplicativo R (R DEVELOPMENT CORE TEAM, 2011)

A diversidade de trepadeiras nas duas florestas foi analisada também pela riqueza de espécies nas árvoressuporte (unidades amostrais), utilizando-se o índice de Shannon-Weaver (H'). Adicionalmente, foi calculado o inverso do índice de Simpson (1/D) (MAGURRAN, 2004).

\section{RESULTADOS}

Nas duas formações foram registrados 177 indivíduos, em 15 famílias, 21 gêneros e 27 espécies. As famílias com maior representatividade em espécies foram Dilleniaceae (cinco), Fabaceae (quatro), Apocynaceae (duas), Combretaceae, Dioscoreaceae, Rubiaceae e Smilacaceae (duas cada). As demais famílias apresentaram apenas um representante. Smilax syphilitica Humb. \& Bonpl. ex Willd. (30), Amphilophium cuneifolium (DC.) L.G. Lohmann (23), Doliocarpus spraguei Cheesman (22), Odontadenia nitida (Vahl) Müll. Arg. (15) e Galactia jussiaeana Kunth (14) se destacaram em número de indivíduos e, juntas, totalizaram $57,8 \%$ do total de indivíduos amostrados. Dez espécies apresentaram apenas um indivíduo amostrado (Tabela 1).

Considerando as formações separadamente, na floresta seca foram registrados 109 indivíduos em 10 famílias, 15 gêneros e 17 espécies. A família mais representativa em número de espécie foi Dilleniaceae (quatro), seguida por Fabaceae, Smilacaceae, Apocynaceae e Rubiaceae (duas espécies cada). As espécies que se destacaram com o maior número de indivíduos foram $S$. syphilitica (26), D. spraguei (22), G. jussiaeana (14) e A. cuneifolium (11). Na floresta inundável, 71 indivíduos em 13 famílias, 16 gêneros e 17 espécies. Dilleniaceae (quatro espécies), Fabaceae (três), Combretaceae e Dioscoreaceae (duas cada) apresentaram o maior número de espécies. A maior abundância foi encontrada em $O$. nitida (15), A. cuneifolium (12) e Doliocarpus amazonicus Sleumer (11).

O número de espécies por formação florestal mostrou que 38\% foram exclusivas da floresta inundável, 31\% da floresta seca e $31 \%$ comuns aos dois ambientes. A análise de MDS não separou as florestas em ambientes distintos pela composição de lianas (Figura 1). A diversidade de Shannon para a floresta inundável foi de $\mathrm{H}^{\prime}=1,08$ e o inverso de Simpson, $1 / \mathrm{D}=8,3$; e para a floresta seca foi de $\mathrm{H}^{\prime}=0,98$ e o inverso de Simpson, $1 / \mathrm{D}=7,2$.

\section{DISCUSSÃO}

Nas florestas brasileiras, a maioria dos trabalhos que investigaram a florística de lianas no Sul (DURIGON et al., 2009) e no Sudeste do Brasil (MORELLATO; LEITÃO-FILHO, 1998; HORA; SOARES, 2002; UDULUTSCH et al., 2004; REZENDE; RANGA, 2005; TIBIRIÇÁ et al., 2006; VILLAGRA; NETO, 2010; CARNEIRO; VIEIRA, 2012) registraram Bignoniaceae e Fabaceae entre as mais ricas, seguidas por Malpighiaceae e Convolvulaceae. Nas florestas estudadas, Fabaceae foi representada por quatro espécies.

Fabaceae e Dilleniaceae, apesar de não serem marcantes na guilda de lianas, apresentaram maior número de espécies com hábito lianescente na Amazônia (AMARAL et al., 2008; JARDIM et al., 2013; QUARESMA; JARDIM, 2014). Fabaceae, por sua vez, é uma das famílias mais diversificadas em todas as regiões fitogeográficas do Brasil e também em todas as regiões tropicais, com estação seca marcante

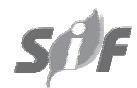

Revista Árvore, Viçosa-MG, v.39, n.2, p.255-261, 2015 
Tabela 1 - Composição florística de lianas em floresta seca (FS) e floresta inundável (FI) da Área de Proteção Ambiental, Algodoal-Maiandeua, Maracanã, Pará, Brasil.

Table 1 - Floristic composition of vines in the dry forest (DF) and flooded forest (FF) in the Environmental Protection Area of Algodal-Maiandeua, Maracanã, Pará State, Brazil.

\begin{tabular}{|c|c|c|c|c|}
\hline Família & Espécie & FS & FI & Total \\
\hline \multirow[t]{2}{*}{ Apocynaceae } & Mandevilla hirsuta (Rich.) K. Schum. & 2 & 0 & 2 \\
\hline & Odontadenia nitida (Vahl) Müll. Arg. & 0 & 15 & 15 \\
\hline Asteraceae & Mikania microphylla Sch. Bip. Ex Baker & 0 & 1 & 1 \\
\hline Acanthaceae & Mendoncia hoffmannseggiana Nees & 0 & 1 & 1 \\
\hline Bignoniaceae & Amphilophium cuneifolium (DC.) L.G. Lohmann & 11 & 12 & 23 \\
\hline \multirow[t]{2}{*}{ Combretaceae } & Combretum laxum Jacq. & 0 & 1 & 1 \\
\hline & Combretum pyramidatum Desv. ex Ham. & 0 & 1 & 1 \\
\hline Convolvulaceae & Maripa reticulata Ducke & 0 & 1 & 1 \\
\hline Celastraceae & Hippocratea volubilis L. & 2 & 0 & 2 \\
\hline \multirow[t]{5}{*}{ Dilleniaceae } & Doliocarpus amazonicus Sleumer & 0 & 11 & 11 \\
\hline & Doliocarpus dentatus (Aubl.) Standl. & 1 & 5 & 6 \\
\hline & Doliocarpus spraguei Cheesman & 22 & 0 & 22 \\
\hline & Davillakunthii A. St.-Hil. & 1 & 4 & 5 \\
\hline & Tetracera willdenowiana Steud. & 9 & 1 & 10 \\
\hline \multirow[t]{2}{*}{ Dioscoreaceae } & Dioscorea ceratandra Uline & 2 & 3 & 5 \\
\hline & Dioscorea trifida L. f. & 0 & 3 & 3 \\
\hline \multirow[t]{4}{*}{ Fabaceae } & Centrosema brasilianum (L.) Benth. & 0 & 1 & 1 \\
\hline & Dioclea bicolor Benth. & 0 & 3 & 3 \\
\hline & Dioclea virgata (Rich.) Amshoff & 3 & 1 & 4 \\
\hline & Galactia jussiaeana Kunth & 14 & 0 & 14 \\
\hline Marantaceae & Ischnosiphon polyphyllus (Poepp. \& Endl.) Körn. & 0 & 1 & 1 \\
\hline Malpighiaceae & Heteropterys nervosa A. Juss. & 8 & 1 & 9 \\
\hline Polygalaceae & Moutabea guianensis Aubl. & 0 & 1 & 1 \\
\hline \multirow[t]{2}{*}{ Rubiaceae } & Chiococca nitida var. amazonica Müll. Arg. & 3 & 0 & 3 \\
\hline & Sabicea amazonensis Wernham & 1 & 0 & 1 \\
\hline \multirow[t]{2}{*}{ Smilacaceae } & Smilax campestris Griseb. & 1 & 0 & 1 \\
\hline & Smilax syphilitica Humb. \& Bonpl. ex Willd. & 26 & 4 & 30 \\
\hline Total & & 109 & 71 & 180 \\
\hline
\end{tabular}

(OLIVEIRA-FILHO; FONTES, 2000; GILLESPIE et al., 2000). O maior tempo de período seco é uma das principais características da área de estudo.

Dilleniaceae apresentou o maior número de espécies e a maior riqueza muito semelhantes ao registrado por Mascaro et al. (2004) na floresta tropical da Estação Biológica de La Selva, na Costa Rica. Esses autores inferiram que a dispersão por pássaros seria a causa da maior abundância dessa família, pois para as lianas a dispersão através de animais é a mais comum em florestas úmidas tropicais (GENTRY, 1991; MASCARO et al., 2004).

O número de espécies lianescentes deste estudo foi considerado baixo quando comparado com as espécies que ocorrem na mata ciliar e na floresta estacional semidecidual no Estado de São Paulo (MORELLATO; LEITÃO-FILHO, 1998), cujos registros foram 102 e 136 espécies, respectivamente. No entanto, assemelhouse à mata de galeria no Rio Grande do Sul, com 27 espécies (BUENO et al., 1987), 23 espécies em uma floresta ombrófila densa (CITADINI-ZANETTE, 1997) e 39 espécies nos morros graníticos (AGUIAR et al., 1986).

A riqueza de lianas é maior em regiões onde a estação seca é prolongada, porque nesse período as árvores perdem parte de sua folhagem e, assim, proporcionam maior penetração de luz, favorecendo o crescimento de plantas escaladoras (VAN DER HEIJDEN; PHILLIPS, 2009; VARGAS et al., 2013). Essa informação está em desacordo com a baixa riqueza

Revista Árvore, Viçosa-MG, v.39, n.2, p.255-261, 2015 
Formações florestais de restinga e relações ecológicas...

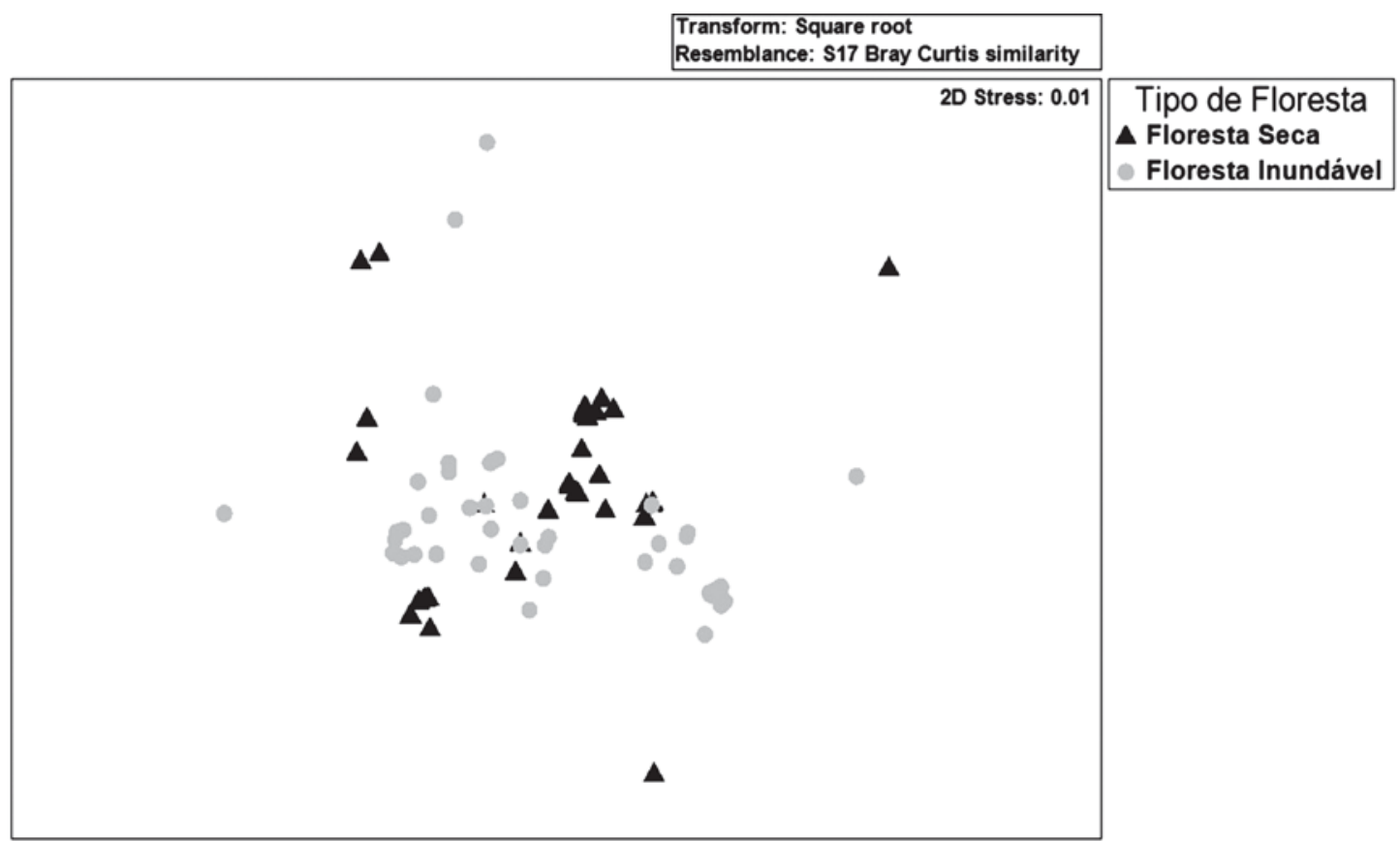

Figura 1 - Análise de escalonamento multidimencional não métrica, mostrando os padrões de agrupamento das unidades amostrais (árvores suporte) na floresta seca (FRS) e floresta inundável (FRI) da Área de Proteção Ambiental Algodoal-Maiandeua, Maracanã, Pará State, Brasil.

Figure 1 - Non-metric multidimensional scaling analysis showing grouping patterns of sample units (support trees) in the dry forest (DF) and flodded forest (FF) in the Environmental Protection Area of Algodoal-Maiandeua, Maracanã, Pará State, Brazil.

encontrada, pois as florestas estudadas situam-se em uma região com estação seca bem definida durante cerca de oito meses e com árvores que não perdem intensivamente suas folhagens.

Dez espécies (37\%) foram amostradas com apenas um representante. Isso, provavelmente, se deve à intensidade de coleta e ao tamanho da área amostral (OLIVEIRA et al., 2008), resultando na baixa representatividade de algumas espécies. A diversidade de espécies na floresta seca e na floresta inundável foi baixa quando comparada com uma floresta ombrófila densa ( $\mathrm{H}^{\prime}=1,69$ nats) (CITADINI-ZANETTE, 1997) e uma floresta estacional semidecidual ( $\mathrm{H}^{\prime}=3,16$ nats) (HORA; SOARES, 2002). Também foi menor que os índices encontrados por Oliveira et al. (2008) para lianas em três ambientes de terra firme na Amazônia Central (Platô, $H^{\prime}=2,50$ nats; Vertente, $H^{\prime}=1,91$ nats; e Baixio, $\mathrm{H}^{\prime}=1,38$ nats $)$.

A diversidade e riqueza de lianas foram maiores em floresta secundária quando comparadas com florestas primárias (DEWALT et al., 2000) e nas bordas da floresta do que no seu interior (LAURENCE et al., 2001). Dessa forma, a baixa diversidade encontrada pode estar relacionada à conservação das florestas estudadas, que são áreas primárias, e também à marcação das parcelas que foram alocadas no interior da floresta.

Smilax syphilitica apresentou o maior número de indivíduos. Isso pode estar relacionado à estratégia de reprodução, pois as espécies desse gênero possuem um sistema caulinar subterrâneo que, através de gemas axilares, se reproduzem assexuadamente, assegurando um estabelecimento bem-sucedido e maior adaptabilidade de sobrevivência ao meio (HOFFMANN, 1998; MEDEIROS; MIRANDA, 2008).

\section{CONCLUSÃO}

Apesar de as florestas apresentarem considerável número de espécies exclusivas (38\% na floresta inundável e $31 \%$ na floresta seca), não houve a formação de grupos pela análise de MDS, evidenciando a não separação dos ambientes através da composição de lianas. 


\section{AGRADECIMENTOS}

Ao Conselho Nacional de Desenvolvimento Científico e Tecnológico, pelo apoio financeiro do projeto pesquisa científica e capacitação local como indicadores sustentáveis para restauração ambiental da flora da APA de Algodoal-Maiandeua, Maracanã, Pará, Brasil. Processo n ${ }^{\circ} 561808 / 2010-4$ e pela Bolsa de Produtividade/CNPq -Processo 305667/2013-0 do segundo autor.

\section{REFERÊNCIAS}

AGUIAR, L.W.;MARTAU, L.; SOARES, Z.F.; BUENO, O.L.; MARIATH, J.E.; KLEIN, R.M. Estudo preliminar da flora e vegetação dos morros graníticos da grande Porto Alegre, Rio Grande do Sul, Brasil. Iheringia, v.34, n.2, p.3-38, 1986 .

AMARAL, D.D.; PROST, M.T.; BASTOS, M.N.C.; COSTA-NETO, S.V.; SANTOS, J.U.M. Restingas do litoral amazônico, estados do Pará e Amapá, Brasil. Boletim do Museu Paraense Emílio Goeldi, Ciências Naturais, v.3, n. 1, p.35-67, 2008.

BRANDES, A.F.N.; BARROS, C.F. Anatomia do lenho de oito espécies de lianas da família Leguminosae ocorrentes na Floresta Atlântica. Acta Botanica Brasilica, v.22, n.2, p.465480, 2008.

BUENO, O.L.; NEVES, M.T.M.B.; OLIVEIRA, M.L.A.A.; RAMOS, R.L.D.; STREHL, T. Florística em áreas da margem direita do baixo Jacuí, RS, Brasil. Acta Botanica Brasilica, v.1, n.2, p.101-121, 1987.

CARNEIRO, J.S.; VIEIRA, A.O.S. Trepadeiras: florística da Estação Ecológica do Caiuá e chave de identificação vegetativa para espécies do Norte do Estado do Paraná. Acta Scientiarum. Biological Sciences, v.34, n.2, p.217-223, 2012.

CARVALHO, P.G.; VAN MELIS, J.MORELLATO, L.P.; GUARANTINI, M.T.G. Critérios para a amostragem de lianas: comparação e estimativa da abundância e biomassa de lianas no cerrado. Revista Árvore, v.37, n.6, p.1037-1043, 2013.
CITADINI-ZANETTE, V.; SOARES, J.J.;

MARTINELLO, C.M. Lianas de um remanescente florestal da microbacia do Rio Novo, Orleans, Santa Catarina Brasil. Ínsula, v.26, n.3, p.45-63, 1997.

DEWALT, S.J.; SCHNITZER, S.A.; DENSLOW, J.S. Density and diversity of lianas along a chronosequence in a central Panamanian lowland forest. Journal of Tropical Ecology, v.16, n.2, p.1-9, 2000.

DURIGON, J.; CONTO-DOROW, T.S.; EISINGER, S.M. Composição florística de trepadeiras ocorrentes em bordas de fragmentos de floresta estacional, Santa Maria, Rio Grande do Sul, Brasil. Rodriguésia, v.60, n.2, p.415-422, 2009.

FREITAS, J.; ASSIS, A.M. Estrutura do componente epífito vascular em trecho de Floresta Atlântica na região serrana do Espírito Santo. Revista Árvore, v.37, n.5, p.815-823, 2013.

GENTRY, A. H. Breeding and dispersal systems of lianas. In: PUTZ, F.E.; MOONEY, H.A. (Eds.). The biology of vines. Cambridge: Cambridge University Press, 1991.p.393-423.

GILLESPIE, T.W.; GRIJALVA, A.; FARRIS, C.N. Diversity, composition, and structure of tropical dry forests in Central America. Plant Ecology, v.147, n.1, p.37-47, 2000.

HOFFMANN, W.A. Post-burn reproduction of woody plants in a neotropical savanna: the relative importance of sexual and vegetative reproduction. Journal of Applied Ecology, v.35, p.422-433, 1998.

HORA, R.C.; SOARES, J.J. Estrutura fitossociológica da comunidade de lianas em uma floresta estacional semidecidual na Fazenda Canchim, São Carlos, SP. Revista Brasileira de Botânica, v.25, n.3, p.323-329, 2002.

JARDIM,D.G.; JARDIM, M.A.G.; QUARESMA, A.C.; COSTA-NETO,S.V. Regeneração natural em formações florestais de uma Unidade de Conservação, Maracanã, Pará, Brasil. Biota Amazônia, v.3, n.3, p.79-87, 2013.

LAURANCE, W.F.; PEREZ-SALICRUP, D.; DELAMÔNICA, P.; FEARNSIDE, P.M.; 
D'ANGELO, S.; JEROZOLINSKI, A.; POHL, L.; LOVEJOY, T.E. Rain forest fragmentation and the structure of Amazonian liana communities. Ecology, v.82, p.105-116, 2001.

MAGURRAN, A.E. Measuring biological diversity. Oxford: Blackwell Science, 2004. $256 \mathrm{p}$.

MASCARO, J.; SCHNITZER, S.A.; CARSON, W.P. Liana diversity, abundance, and mortality in a tropical wet forest in Costa Rica. Forest Ecology and Management, v.190, p.3-14, 2004.

MEDEIROS, M.B.; MIRANDA, H.S. Post-fire resprouting and mortality in cerrado woody plant species over a three-year period. Edinburg Journal of Botany, v.65, n.1, p.53-68, 2008.

MORELLATO, L.P.; LEITÃO-FILHO, H.F. Levantamento florístico da comunidade de trepadeiras de uma floresta semidecídua no Sudeste do Brasil. Boletim do Museu Nacional, Série Botânica, v.103, p.1-15, 1998.

OLIVEIRA, A.N.; AMARAL, I.L.; RAMOS; M.B.P.; FORMIGA, K.M. Aspectos florísticos e ecológicos de grandes lianas em três ambientes florestais de terra firme na Amazônia Central. Acta Amazonica, v.38, n.2, p.421-430, 2008.

OLIVEIRA-FILHO, A.T.; FONTES, M.A.L. Patterns of floristic differentiation among Atlantic forests in southeastern Brazil and the influence of climate. Biotropica, v.32, p.793$810,2000$.

QUARESMA, A.C.; JARDIM, M.A.G. Floristic composition and spatial distribution of vascular epiphytes in the restingas of Maracanã, Brazil.

Acta Botanica Brasilica, v.28, n.1, p.68-75, 2014.
REZENDE, A.A.; RANGA, N.T. Lianas da Estação Ecológica do Noroeste Paulista, São José do Rio Preto/Mirassol, SP, Brasil. Acta Botanica Brasilica, v.19, n.2, p.273-279, 2005.

R DEVELOPMENT CORE TEAM. R: A language and environment for statistical computing. R Foudation for statistical Computing, Vienna. 2011.http://www.R-project.org/.

SCHMITZER, S.A.; BONGERS, F. The ecology of lianas and their role in forests. Trends in Ecology \& Evolution, v.17, p.223-230, 2002.

TIBIRIÇÁ, Y.J.A.; COELHO, L.F.M.; MOURA, L.C. Florística de lianas em um fragmento de floresta estacional semidecidual, Parque Estadual de Vassununga, Santa Rita do Passa Quatro, SP, Brasil. Acta Botanica Brasilica, v.20, n.2, p.339-346, 2006.

UDULUTSCH, R.G.; ASSIS, M.A.; PICCHI, D.G. Florística de trepadeiras numa floresta estacional semidecídua, Rio Claro - Araras, Estado de São Paulo, Brasil. Revista Brasileira de Botânica, v.27, n.2, p.125-134, 2004.

van der HEIJDEN, G.M.F.; PHILLIPS, O.L. Liana infestation impacts tree growth in a lowland tropical moist forest. Biogeosciences, v.6, p.2217-2226, 2009.

VARGAS, B.C.; ARAÚJO, G.M.; SCHIAVINI, I.; ROSA, P.O.; HATTORI, E.K.O. Florística de trepadeiras em Floresta Semidecidual e em Mata Ciliar no vale do rio Araguari, MG. Bioscience Journal, v.29, p.185-197, 2013.

VILLAGRA, B.L.P.; ROMANIUC NETO, S. Florística de trepadeiras no Parque Estadual das Fontes do Ipiranga, São Paulo, SP, Brasil. Revista Brasileira de Biociências, v.8, n.2, p.186-200, 2010. 